\title{
The Hippocratic Splash
}

\author{
Geetha Girithari ${ }^{1}$, Inês Coelho dos Santos ${ }^{1}$, Eva Claro ${ }^{1}$, Serguey Belykh², David Matias ${ }^{2}$, Orlando Santos ${ }^{3}$ \\ ${ }^{1}$ Department of Internal Medicine, Centro Hospitalar do Médio Tejo, Abrantes, Portugal \\ ${ }^{2}$ Department of Emergency Medicine, Centro Hospitalar do Médio Tejo, Abrantes, Portugal \\ ${ }^{3}$ Department of Pneumology, Centro Hospitalar do Médio Tejo, Abrantes, Portugal
}

Received: 06/10/2018

Accepted: $15 / 10 / 2018$

Published: 13/11/2018

How to cite this article: Girithari G, Coelho dos Santos I, Claro E, Belykh S, Matias D, Santos O. The hippocratic splash. EJCRIM 2018;5: doi:10.12890/2018_000975.

Conflicts of Interests: The Authors declare that there are no competing interests.

This article is licensed under a Commons Attribution Non-Commercial 4.0 License

\section{ABSTRACT}

Although the current medical literature is limited, hydropneumothorax was described as far back as the 5 th century BC. It is characterized by the presence of air and fluid in the pleural cavity and is an infrequent finding. Causes include trauma, iatrogenesis following thoracentesis, the presence of gas-forming organisms, tuberculosis and malignancy. Diagnosis is based on clinical and radiological features. We report a case of hydropneumothorax and present radiological images showing the distinctive features of this entity.

\section{LEARNING POINTS}

- Hydropneumothorax is defined as the presence of air and fluid in the pleural cavity and is an infrequent finding.

- Clinical features may present as breathlessness and chest pains with decreased breath sounds, dullness in a straight line, shifting dullness, a succussion splash and a positive coin test on physical examination; supine radiography demonstrates a distinctive pleural line with increased density lateral in the pleural cavity.

- Hydropneumothorax is managed by chest tube insertion for intercostal drainage.

\section{KEYWORDS}

Hydropneumothorax, pneumothorax, radiology, Mycobacterium xenopi

\section{INTRODUCTION}

Hydropneumothorax is characterized by the presence of air and fluid in the pleural cavity ${ }^{[1]}$. Current medical literature is scarce although this clinical entity was documented in the 19th century; indeed, the Hippocratic succussion splash was used in ancient Greek medicine in the 5 th century $\mathrm{BC}^{[1,2]}$. Hydropneumothorax is an infrequent presentation in the emergency room (ER) but can be life threatening. Our case highlights the importance of clinical and radiological recognition of this medical emergency.

\section{CASE REPORT}

A 58-year-old man with a recent diagnosis of meningioma awaiting surgical intervention with a history of chronic alcohol and tobacco (80 pack-years) use, was admitted to the pneumology department of our district hospital for a respiratory infection and on-going investigation of multiple cavitated lesions that were mostly present on the left side of his lung. He responded well to antibiotics (amoxicillin and clavulanic acid, clarithromycin) and was discharged with an inconclusive diagnosis with appointments for follow-up consultations for further monitoring and investigation. 
Two months later, he presented at the emergency room (ER) with a 3-day history of fatigue and breathlessness with progressive worsening of his symptoms. He also noted coughing up small quantities of whitish mucus and denied having chest pains, fever or bloody expectorations. Clinical examination revealed a blood pressure of $125 / 93 \mathrm{mmHg}$, tachycardia of 111 beats per minute and slight tachypnoea with peripheral blood saturation of $95 \%$ at room air. Examination of the respiratory system revealed absent breath sound in the left hemithorax with tympanic and dull percussion notes on the superior and inferior halves of the lung, respectively. Succussion splash and shifting dullness were present. Routine blood analysis showed haemoglobin of $8.0 \mathrm{~g} / \mathrm{dl}$, white blood cell counts of $23.58 \times 10^{9}$ cells per litre, neutrophils of $74.5 \%$, lymphocytes of $7.8 \%$, glucose of $116 \mathrm{mg} / \mathrm{dl}$ and C-reactive protein of $2.91 \mathrm{mg} / \mathrm{dl}$. Blood urea and creatinine were normal. Arterial blood gas (ABG) without supplementary oxygen demonstrated hypoxaemia and slight hyperlactataemia $\left(\mathrm{pH}^{7.43}, \mathrm{pO}_{2} 66 \mathrm{mmHg}, \mathrm{pCO}_{2} 39\right.$ $\mathrm{mmHg}, \mathrm{SpO}_{2} 93 \%, \mathrm{HCO}_{3} 25.9 \mathrm{mmol} / \mathrm{l}, \mathrm{BE} 1.6 \mathrm{mmol} / \mathrm{l}$, lactate $\left.1.6 \mathrm{mmol} / \mathrm{l}\right)$. Sputum bacterial and fungus cultures were normal. Sputum smear for acid-fast bacilli was negative.

Chest radiography was ordered and demonstrated a left-sided hydropneumothorax with contralateral deviation of the left mediastinum and the presence of multiple thin-walled cavitated lesions on the right side (Fig. 1). Chest tube thoracostomy was performed. Pleural fluid was drained and cytology indicated mononuclear predominance while cultures were negative for bacteria, fungus and Koch's bacillus. A computed tomography (CT) scan of the chest confirmed hydropneumothorax on the left side of the lung, not loculated, with a maximum thickness of $4 \mathrm{~cm}$, resulting in slight contralateral deviation of the mediastinum, bilateral multiple cavitated lesions and irregular densities on the base of the left lung suggesting atelectasis (Fig. 2).

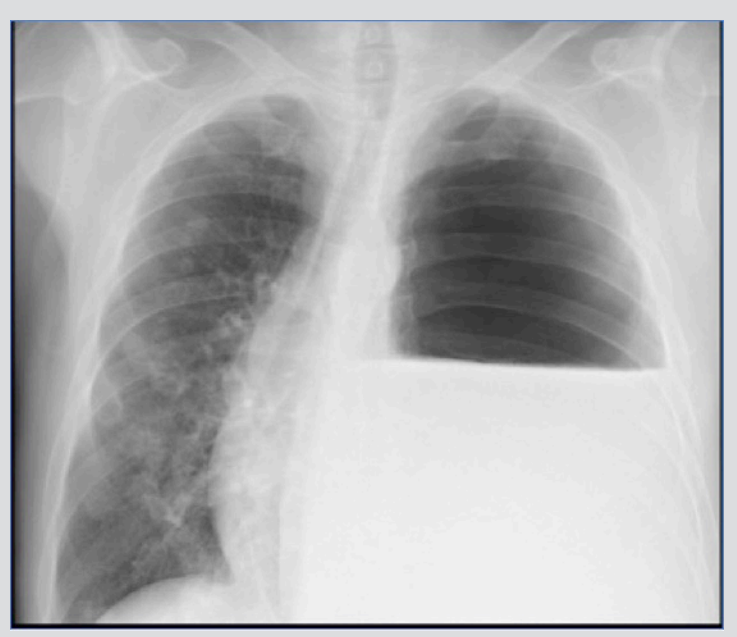

Figure 1. Chest radiography showing left-sided hydropneumothorax with contralateral deviation of the left mediastinum and the presence of multiple thin-walled cavitated lesions on the right side

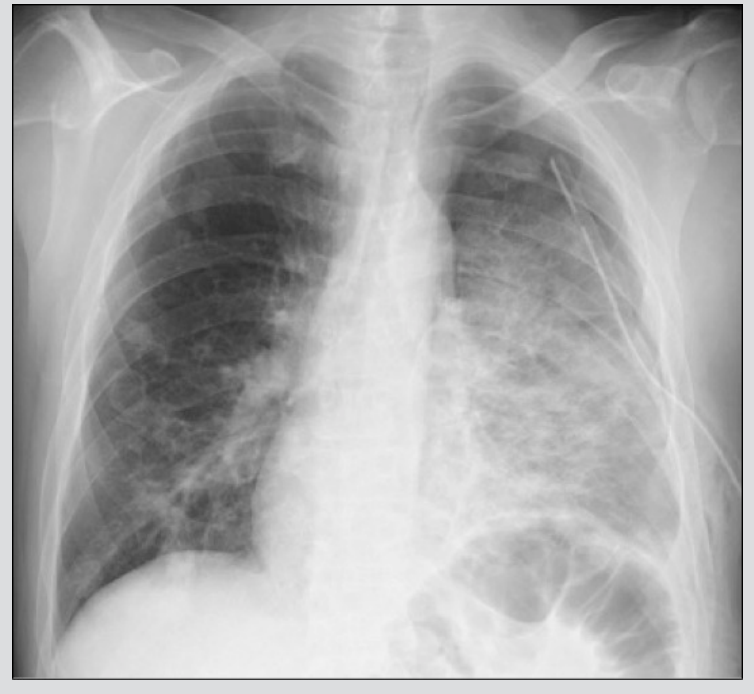

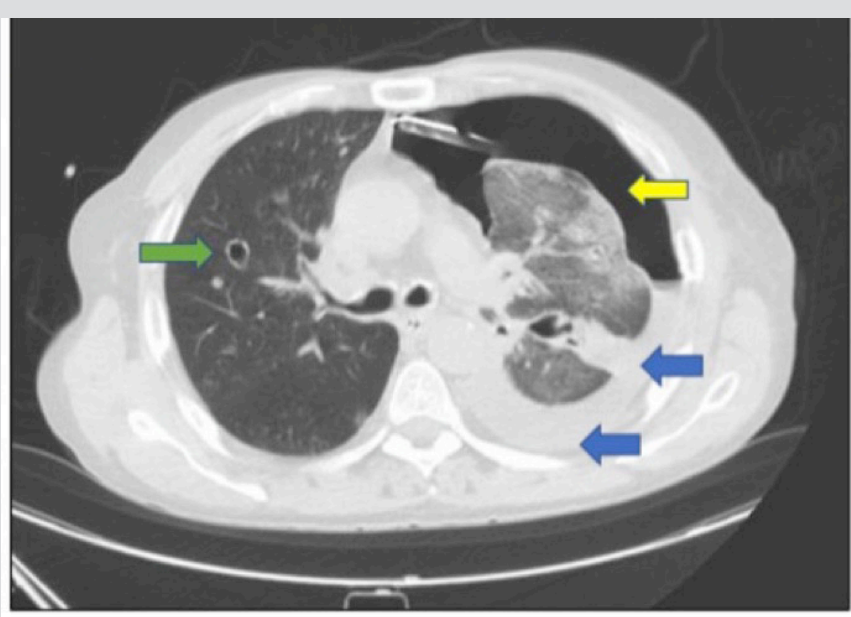

Figure 2. Computed tomography (CT) showed hydropneumothorax on the left side of the lung, not loculated, with a maximum thickness of $4 \mathrm{~cm}$ (blue arrows), resulting slight contralateral deviation of the mediastinum, a cavitated lesion (green arrow) and irregular densities on the base
Figure 3. Post-drainage chest radiography showed re-expansion of the left lung with significant reduction of the hydropneumothorax and resolution of the deviated mediastinum. Left hemi-diaphragmatic elevation suggests a collapsed left lung 
The thoracostomy tube was positioned in the anterior middle third of the left pleural cavity and drained $3000 \mathrm{ml}$ of serohaematic fluid within 24 hours. A repeat chest radiograph showed re-expansion of the left lung with a significant reduction of the hydropneumothorax and resolution of the deviated mediastinum (Fig. 3). A total of $4995 \mathrm{ml}$ of fluid was drained over 3 days. Blood cultures and an HIV test were negative, but Middlebrook 7H9 Broth was positive for Mycobacterium xenopi.

The patient was transferred to the cardio-thoracic department of a central hospital for further investigation.

\section{DISCUSSION}

Common causes of hydropneumothorax include trauma, iatrogenesis following thoracentesis and the presence of gas-forming organisms which are normally rare ${ }^{[3]}$. Other causes include tuberculosis and malignancy ${ }^{[1]}$.

In our patient, the exact cause of the hydropneumothorax has yet to be determined. The incidence of pulmonary diseases associated with non-tuberculous mycobacteria such as $M$. xenopi has been rising and is associated with a high rate of pneumothorax ${ }^{[4]}$. To the best of our knowledge, the literature does not mention M. xenopi as a cause of hydropneumothorax although it does not rule it out. All possible causes of hydropneumothorax must be investigated, especially in the context of malignancy given that our patient has a history of meningioma (a small number of meningiomas can be cancerous), and primary or secondary oncological lesions should be confirmed or ruled-out.

In the ER setting, physicians should be alert to clinical symptoms and signs of hydropneumothorax. Breathlessness followed by chest pains are the most common presenting symptoms in these patients. Fever, cough and constitutional symptoms such as weight loss and anorexia could be due to other specific aetiologies such as infection or malignancy ${ }^{[1]}$. Physical examination demonstrates significantly decreased or abolished breath sounds, dullness in a straight line (over the fluid), shifting dullness, a succussion splash and a positive coin test ${ }^{[5]}$.

The diagnosis of hydropneumothorax is based on the detection of a pleural line with increased density lateral in the pleural space seen on supine radiography. This is important in light of therapeutic tube thoracostomy placement ${ }^{[6]}$.

Chest drainage is the appropriate management for hydropneumothorax. Supporting treatment should be according to aetiology. Future research is vital for the development of suitable therapeutic management of hydropneumothorax.

\section{REFERENCES}

1. Awad N, Kasargod V. Clinical profile, etiology, and management of hydropneumothorax: an Indian experience. Lung India 2016;33:278.

2. Thompson H. Clinical lecture on a case of hydropneumothorax. BMJ 1872;2:373-375.

3. Reed A, Dent M, Lewis S, Shogun P, Folio L. Hydropneumothorax verses simple pneumothorax. Military Medicine Radiology Corner 2010;175.

4. Ueyama M, Asakura T, Morimoto K, Namkoong H, Matsuda S, Osawa T, et al. Pneumothorax associated with nontuberculous mycobacteria. Medicine (Baltimore) 2016;95:e4246.

5. Sarkar M, Madabhavi I, Niranjan N, Dogra M. Auscultation of the respiratory system. Ann Thorac Med 2015;10:158-168.

6. Onik G, Goodman PC, Webb WR, Brasch RC. Hydropneumothorax: detection on supine radiographs. Radiology 1984;152:31-4. 